\title{
Development and Evaluation of Isothermal Amplification Methods for Rapid Detection of Lethal Amanita Species
}

\author{
Zhengmi He, Yuting Su, Sainan Li, Pan Long, Ping Zhang and Zuohong Chen* \\ College of Life Sciences, Hunan Normal University, Changsha, China
}

In the present work, loop-mediated isothermal amplification (LAMP) and hyperbranched rolling circle amplification (HRCA) methods were developed to detect and distinguish different lethal Amanita species. Specific LAMP primers and HRCA padlock probes for species-specific identification and a set of universal LAMP primers for lethal Amanita species were designed and tested. The results indicated that the LAMPbased assay was able to discriminate introclade lethal Amanita species but was not able to discriminate intraclade species perfectly, while the HRCA-based assay could discriminate whether introclade or intraclade species. The universal LAMP primers were positive for 10 lethal species of Amanita section Phalloideae and negative for 16 species of Amanita outside section Phalloideae. The detection limits of LMAP and HRCA were 10 and $1 \mathrm{pg}$ of genomic DNA per reaction, respectively. In conclusion, the two methods could be rapid, specific, sensitive and low-cost tools for the identification of lethal Amanita species.

Keywords: loop-mediated isothermal amplification, hyperbranched rolling circle amplification, ITS sequence, lethal amanitas, padlock probe

\section{INTRODUCTION}

Mushroom poisoning is the main cause of mortality in food poisoning incidents in China. According to the National Management Information System of Public Health Emergency, in China, 576 mushroom poisoning events were reported from 2004 to 2014, with 3701 poisoning cases and 786 deaths; the fatality rate of mushroom poisoning accounted for $35.57 \%$ of total food poisoning (2210) deaths (Zhou et al., 2016). More than 90\% of fatal mushroom poisoning cases were caused by mistaken ingestion of lethal amanitas in Europe, North America and East Asia (Enjalbert et al., 2002; Chen et al., 2014). Lethal amanitas are a group of cyclopeptide-containing mushrooms classified in genus Amanita section Phalloideae (Fr.) Quél. (Cai et al., 2014; Tang et al., 2016). There have been approximately 50 lethal Amanita species reported worldwide (Cai et al., 2016). These lethal Amanita species have four common morphologic characteristics as bases distinguished from other taxa of Amanita, including a non-appendiculate pileus, the persistent presence of an annulus, a bulbous stipe base with a limate volva and amyloid basidiospores (Cai et al., 2014). The containing substances of various peptide toxins were another critical characteristics of lethal amanitas and the peptide toxins in Amanita can be divided into three major groups, including amatoxins, phallotoxins and virotoxins, which are bicyclic octapeptides, bicyclic heptapeptides and 
monocyclic heptapeptides, respectively (Wieland, 1986). The primary toxins responsible for fatal human poisoning of these lethal Amanita species are amatoxins that induce acute liver failure through binding with eukaryotic DNAdependent RNA polymerase II and subsequently inhibiting the elongation essential to transcription (Walton, 2018). In wild, some lethal Amanita species are similar to the edible species of section Caesareae Singer, for example, A. chepangiana (edible) vs. A. exitialis (lethal) and A. hemibapha (edible) vs. A. subjunquillea (lethal), this is the main reason for mistaken collection and ingestion.

Rapid identification of poisonous mushroom species eaten by patients is very important for toxic source investigation, clinical diagnosis and proper treatment. Hence, the establishment of rapid and effective methods for detection of lethal amanitas is urgently needed. To date, the identification and detection methods for lethal Amanita species mainly depend on their morphological and anatomical evidence, toxin analysis and molecular methods, such as PCR amplification and sequencing of DNA barcoding (Enjalbert et al., 1992; Epis et al., 2010; Harper et al., 2011). However, these methods were often time consuming and complicated and dependent on expensive equipment and professionals, which were difficult to implement in primary institutions or remote areas. Thus, development of simple, rapid and low-cost detection methods would be helpful in curing mushroom poisoning at its early onset as well as investigating the toxin.

In recent years, loop-mediated isothermal amplification (LAMP) and hyperbranched rolling circle amplification (HRCA) have been widely used for molecular detection and identification of pathogenic fungi (Niessen and Vogel, 2010; Tsui et al., 2010; Dai et al., 2012; Davari et al., 2012; Duan et al., 2014; Trilles et al., 2014), in view of their rapid and sensitive detection in addition to the wide range of detection strategies available and the ability of the technique to be deployed outside conventional laboratory settings. LAMP requires a set of four primers (FIP, BIP, F3, and B3) aimed at the six different specific regions of target DNA, and the reaction happens at a constant temperature $\left(60-65^{\circ} \mathrm{C}\right)$ catalyzed by Bst DNA polymerase (Notomi et al., 2000). A vast number of products $\left(10^{9}-10^{10}\right.$-fold) with a dumbbell structure, which are formed by strand displacement of the outer and inner primers, are produced by cycle amplification. The reaction time is generally about an hour, but if loop primers are added, the time consumed will be shorten by half (Nagamine et al., 2002). Unlike LAMP, the HRCA employs a linear padlock probe that hybridizes with a target DNA and is then ligated by DNA ligase to form a circular probe, which subsequently serves as the template to proceed as a turn-by-turn cascade of multiple hybridization, primer extension, and strand displacement involving two primers under isothermal conditions and finally a $>10^{9}$-fold amplification of products is generated from the reaction (Nilsson et al., 1994; Lizardi et al., 1998).

To date, as far as we know, only one report has been published about the LAMP-based method for rapid mushroom species identification (Vaagt et al., 2013). In this paper, the LAMP assays were used for the rapid and easy detection of the death cap mushroom Amanita phalloides from closely related edible and toxic mushroom species. Because there have been many lethal Amanita species and similarities among these species, the aims of this study are (i) to develop LAMP and HRCA methods for species-specific identification of lethal Amanita species and (ii) to design specific but universal LAMP primers for identification of all lethal Amanita species.

\section{MATERIALS AND METHODS}

\section{Mushroom Samples and Identification}

A total of 26 Amanita mushroom species were used in this study, and their information is listed in Table 1, including 10 lethal species in Amanita section Phalloideae (Supplementary Figure S1) and 16 species of Amanita outside section Phalloideae. Among them, the Amanita phalloides samples were provided by Professor Li TH (Guangdong Institute of Microbiology, China), which were collected from Lazio, Rome, Italy, in October 2014; the A. bisporigera samples were collected by Zhang from Hamilton, Canada, in August 2009; and the remaining 24 tested mushroom samples were collected from China. All the mushroom materials were identified by both morphological and molecular evidence (ITS sequence) following Zhang et al. (2010) and Cai et al. (2014). The samples determined in this study were deposited in Mycological Herbarium of Hunan

\begin{tabular}{|c|c|c|c|}
\hline Amanita section & Species & Specimen no. & $\begin{array}{l}\text { GenBank } \\
\text { accession no. }\end{array}$ \\
\hline \multirow[t]{10}{*}{ Sect. Phalloideae } & A. bisporigera & MHHNU 7224 & KU311692 \\
\hline & A. exitialis & MHHNU 30297 & KT003192 \\
\hline & A. fuliginea & MHHNU 30944 & KU356798 \\
\hline & A. pallidorosea & MHHNU 8112 & KU311697 \\
\hline & A. phalloides & GDGM 41101 & KT003193 \\
\hline & A. rimosa & MHHNU 7954 & KU311695 \\
\hline & A. subfuliginea & MHHNU 8812 & $\mathrm{MH} 142183$ \\
\hline & A. subjunquillea & MHHNU 7751 & KR996715 \\
\hline & A. subpallidorosea & MHHNU 8617 & KU601411 \\
\hline & A. virosa & MHHNU 8621 & KY472227 \\
\hline \multirow[t]{4}{*}{ Sect. Amanita } & A. rubrovolvata & MHHNU 8591 & KU356797 \\
\hline & A. rufoferruginea & MHHNU 30943 & KU497532 \\
\hline & A. sinensis & MHHNU 8585 & KU497533 \\
\hline & A. sychnopyramis & MHHNU 30253 & KU497534 \\
\hline Sect. Caesareae & A. javanica & MHHNU 30270 & KU497535 \\
\hline \multirow[t]{3}{*}{ Sect. Vaginatae } & A. fulva & MHHNU 8550 & KU497536 \\
\hline & A. orientifulva & MHHNU 8580 & KU497537 \\
\hline & A. vaginata & MHHNU 30266 & KU497538 \\
\hline Sect. Amidella & A. neoovoidea & MHHNU 30952 & KU497539 \\
\hline \multirow[t]{3}{*}{ Sect. Lepidella } & A. kotohiraensis & MHHNU 30259 & KU497540 \\
\hline & A. oberwinklerana & MHHNU 30819 & KT003191 \\
\hline & A. pseudoporphyria & MHHNU 30897 & KU497541 \\
\hline \multirow[t]{4}{*}{ Sect. Validae } & A. citrina & MHHNU 30252 & KU497542 \\
\hline & A. orsonii & MHHNU 8562 & KU497543 \\
\hline & A. sepiacea & MHHNU 8474 & KU497544 \\
\hline & A. spissacea & MHHNU 8472 & KU497545 \\
\hline
\end{tabular}




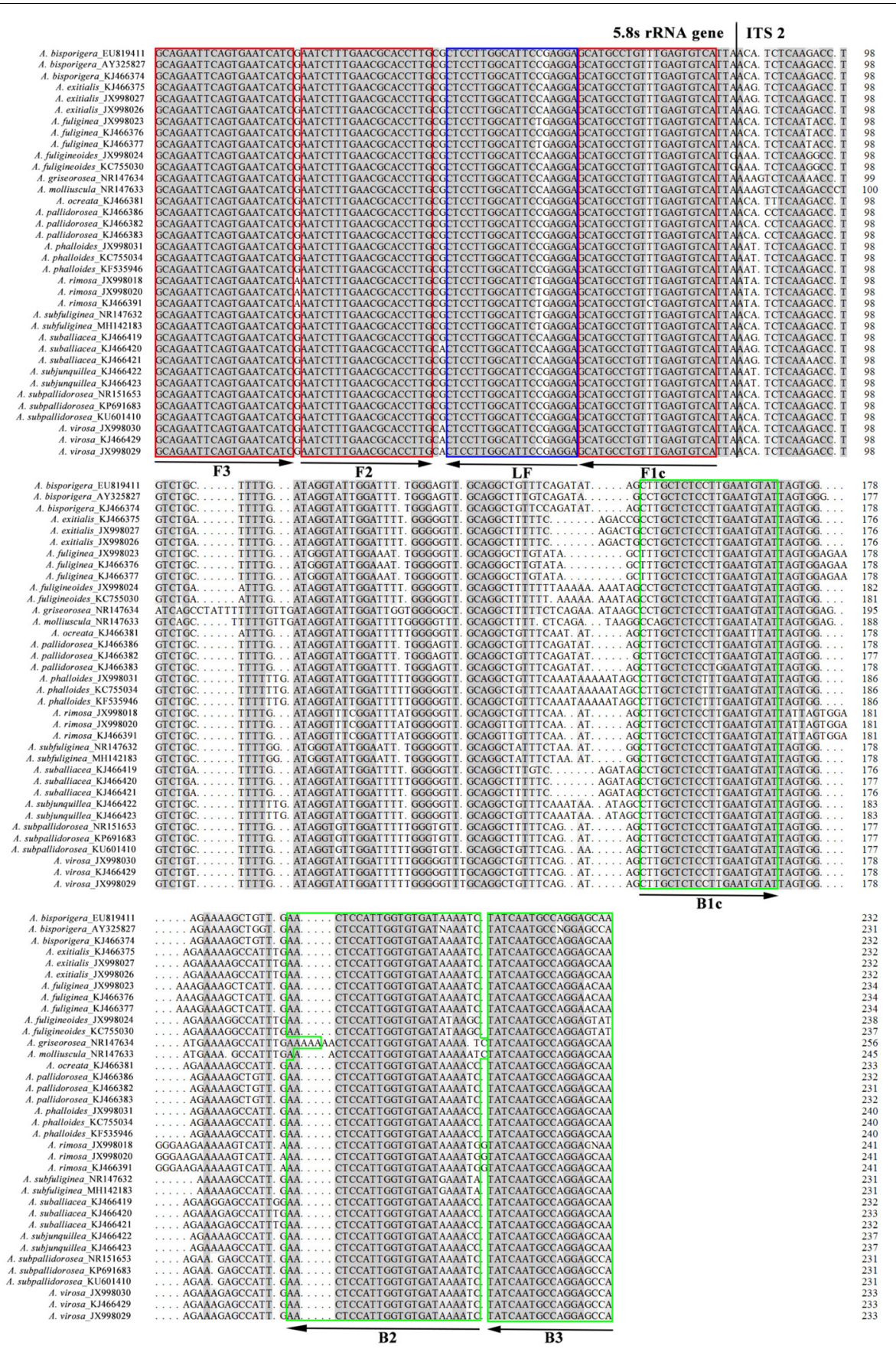

FIGURE 1 | Multiple alignment of ITS sequences of fifteen lethal Amanita species. The target regions used for designing the universal primers were labeled with boxes and arrows, and the aligned sequences were partial 5.8S ribosomal RNA gene and internal transcribed spacer 2 .

Normal University (MHHNU) and Mycological Herbarium of Guangdong Institute of Microbiology (GDGM).

\section{DNA Extraction, PCR Amplification and Sequencing}

Total genomic DNA was extracted by the Fungal DNA Mini Kit (OMEGA, United States) and then diluted to $10 \mathrm{ng} / \mu \mathrm{L}$ as a working concentration. The primers ITS 4 and ITS 5 were used for amplification of ITS sequences. The PCR mixtures contained $1 \times$ PCR buffer, $1.5 \mathrm{mM} \mathrm{MgCl} 2,0.2 \mathrm{mM}$ dNTPs, $0.4 \mu \mathrm{M}$ of each primer, $1.25 \mathrm{U}$ of Taq polymerase, and $1 \mu \mathrm{L}$ DNA template in a total volume of $25 \mu \mathrm{L}$. PCR was performed with an Eppendorf Mastercycler thermal cycler (Eppendorf Inc., Germany) as follows: initial denaturation at $94^{\circ} \mathrm{C}$ for $4 \mathrm{~min}$, followed by 30 cycles of $94^{\circ} \mathrm{C}$ for $30 \mathrm{~s}, 54^{\circ} \mathrm{C}$ for $30 \mathrm{~s}, 72^{\circ} \mathrm{C}$ 
for $30 \mathrm{~s}$, and a final extension at $72^{\circ} \mathrm{C}$ for $8 \mathrm{~min}$. Amplified PCR products were detected by gel electrophoresis on a $1 \%$ agarose gel and then sent to Tsingke Biological Technology (China) for sequencing.

\section{Phylogenetic Tree Building}

Ten ITS sequences of lethal amanitas were obtained by sequencing in this study, and twenty-seven ITS sequences from GenBank were aligned by Clustal X 2.0 software (Larkin et al., 2007). Then, the alignment data of these sequences were used to construct a maximum likelihood phylogeny tree with 1000 bootstrap replicates using MEGA 6.0 software (Tamura et al., 2013).

\section{Primers and Padlock Probes Design}

ITS sequences were chosen as the candidate targets for LAMP primer and padlock probe (PLP) design. For the design, 27 ITS sequences of $A$. bisporigera, A. exitialis, A. fuliginea, A. pallidorosea, A. phalloides, A. rimosa, A. subfuliginea, A. subjunquillea, A. subpallidorosea and $A$. virosa were downloaded from NCBI GenBank and were compared and aligned using DNAMAN 7.0 software to find different target recognition regions for each species and to identify informative nucleotide polymorphic sites conserved within a single species but divergent among different species.

The ten sets of specific LAMP primers were designed by using PrimerExplorer $\mathrm{V}^{1}$. In addition, a set of universal primers for lethal amanitas were manually designed based on the multiple alignment of thirty-six published ITS sequences of fifteen lethal Amanita species. A forward inner primer (FIP) consisted of the complementary sequence of F1c and F2, a backward inner primer (BIP) consisted of B1C and B2, two outer primers (F3 and B3). Loop primers (LF or LB) were used for LAMP, and the structure of the universal primers and their complementarity to target DNA are exemplified in Figure 1.

The ten specific PLPs were designed according to criteria as previously described by Kaocharoen et al. (2008) and Lackner et al. (2012). The PLP consists of two terminal regions complementary to a target sequence located at both ends and a linker region in the middle, which was a partial sequence of the inactive $\mathrm{X}$ specific transcripts (Xist) gene of Mus musculus but lacked homology for the target genes (Figure 2). To ensure the efficiency of padlock probe binding, the padlock probes were predicted with MFOLD to ensure the minimal secondary structure and were designed with the $5^{\prime}$-end probe binding arm $\operatorname{Tm}\left(62-66^{\circ} \mathrm{C}\right)$ close to or above the ligation temperature $\left(65^{\circ} \mathrm{C}\right.$ in this study, see below). To increase $3^{\prime}$-end binding specificity, the $3^{\prime}$ end probe binding arm was designed with a $\operatorname{Tm}\left(45-48^{\circ} \mathrm{C}\right)$ $10-15^{\circ} \mathrm{C}$ below ligation temperature. The $5^{\prime}$ terminal end of the PLP was modified by phosphorylation to allow ligation. In addition, the HRCA primers (HRCA-primer 1, 2), which are used to amplify the specific padlock probe signal during HRCA, were specifically designed to bind to the flanking linker regions of the above-designed padlock probes (Figure 2).

${ }^{1}$ http://primerexplorer.jp/lampv5e/index.html

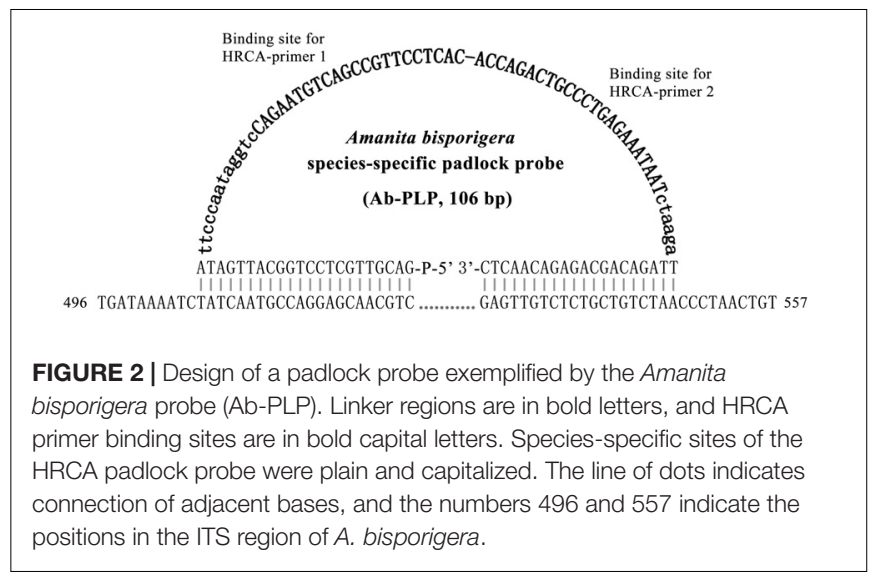

The primers (PAGE) and PLPs (HPLC) were synthesized by Tsingke Biological Technology (China), and their detailed sequences and lengths are shown in Table 2.

\section{LAMP Reaction and Product Detection}

The LAMP reaction was carried out in $10 \mu \mathrm{L}$ reaction mixtures: $1 \times$ ThermoPol buffer $\left(20 \mathrm{mM}\right.$ Tris- $\mathrm{HCl}, 10 \mathrm{mM}\left(\mathrm{NH}_{4}\right)_{2} \mathrm{SO}_{4}$, $10 \mathrm{mM} \mathrm{KCl,} 2 \mathrm{mM} \mathrm{MgSO} 4,0.1 \%$ Triton X-100, PH 8.8), 4 mM $\mathrm{MgSO}_{4}, 1.4 \mathrm{mM}$ dNTP mix, $1.28 \mu \mathrm{M}$ FIP, $1.28 \mu \mathrm{M}$ BIP, $0.12 \mu \mathrm{M}$ F3, $0.12 \mu \mathrm{M}$ B3, 3.2 U Bst DNA polymerase (NEB, United States), $1 \mu \mathrm{L}$ DNA template (10 ng), $150 \mu \mathrm{M} \mathrm{HNB}$, and $\mathrm{ddH}_{2} \mathrm{O}$ to $10 \mu \mathrm{L}$. The reaction was performed in a $0.2 \mathrm{~mL}$ tube with a water bath incubated at $62^{\circ} \mathrm{C}$ for $60 \mathrm{~min}$ and finally $80^{\circ} \mathrm{C}$ for 10 min to termination.

Two approaches were used to analyze DNA amplification, including direct visual inspection of the color of the LAMP mixture with HNB dye and $2 \%$ agarose gel electrophoresis.

\section{HRCA Reaction and Product Detection}

The ligation was carried out in a $10 \mu \mathrm{L}$ mixture containing: $1 \times$ Taq DNA ligase buffer $(20 \mathrm{mM}$ Tris- $\mathrm{HCl}, 25 \mathrm{mM} \mathrm{KAc}$, $10 \mathrm{mM} \mathrm{Mg}(\mathrm{Ac})_{2}, 10 \mathrm{mM}$ DTT, $1 \mathrm{mM}$ NAD, 0.1\% Triton X-100), 10 pM linear padlock probe, $12 \mathrm{U}$ of Taq DNA ligase (NEB, United States) and $1 \mu \mathrm{L}$ of DNA template $(10 \mathrm{ng})$. The ligation mixture was incubated at $65^{\circ} \mathrm{C}$ for $1 \mathrm{~h}$.

After ligation, $1 \mu \mathrm{L}$ of ligation product was added into an HRCA reaction mixture containing $1 \times$ ThermoPol buffer (20 mM Tris- $\mathrm{HCl}, 10 \mathrm{mM}\left(\mathrm{NH}_{4}\right)_{2} \mathrm{SO}_{4}, 10 \mathrm{mM} \mathrm{KCl}, 2 \mathrm{mM}$ $\mathrm{MgSO}_{4}, 0.1 \%$ Triton X-100), $0.4 \mathrm{mM}$ dNTP mix, $0.5 \mu \mathrm{M}$ of each HRCA primers and $1.6 \mathrm{U}$ Bst DNA polymerase (NEB, United States) with a total $10 \mu \mathrm{L}$ volume. The reaction was performed in a $0.2 \mathrm{~mL}$ tube in a water bath incubated at $62^{\circ} \mathrm{C}$ for $60 \mathrm{~min}$.

The results were judged by the appearance of color after adding $1 \mu \mathrm{l}$ of $1000 \times$ SYBR Green I dye to the system after the reaction or $2 \%$ agarose gel electrophoresis of the HRCA product.

\section{Specificity of LAMP Primers and HRCA PLPs}

To test the specificity of the ten sets of specific LAMP primers and HRCA PLPs designed above, genomic DNA 
TABLE 2 | The information of primers and padlocks used for amplification.

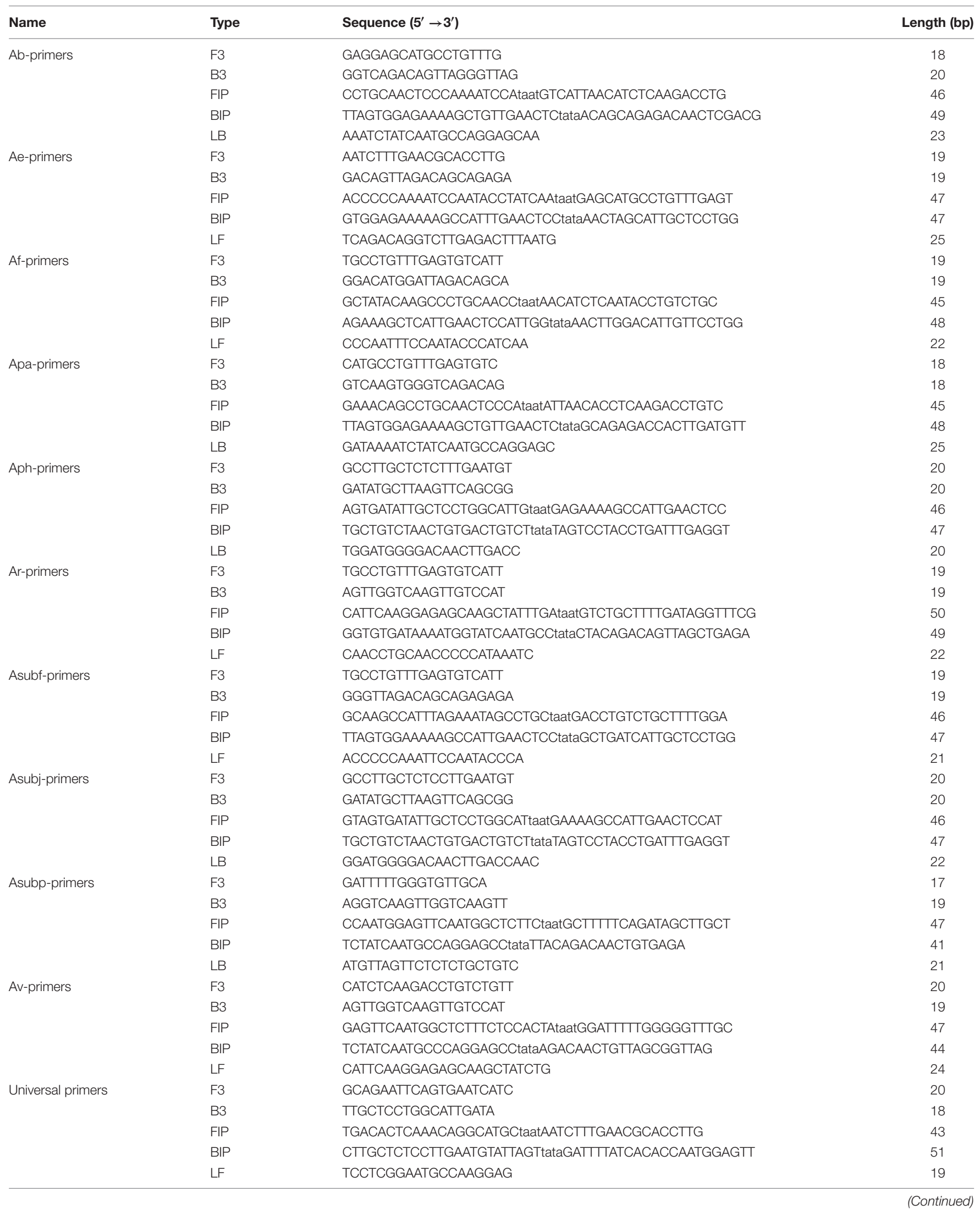


TABLE 2 | Continued

\begin{tabular}{|c|c|c|c|}
\hline Name & Type & Sequence $\left(5^{\prime} \rightarrow 3^{\prime}\right)$ & Length (bp) \\
\hline Ab-PLP & & $\begin{array}{l}\text { P-GACGTTGCTCCTGGCATTGATATTCCCAATAGGTCCAGAATGTCAGCCGTTCCT } \\
\text { CACACCAGACTGCCCTGAGAAATAATCTAAGATTAGACAGCAGAGACAACTC }\end{array}$ & 106 \\
\hline Ae-PLP & & $\begin{array}{l}\text { P-CATTGCTCCTGGCATTGATAGATTTTCCCAATAGGTCCAGAATGTCAGCCGTTCCTC } \\
\text { ACACCAGACTGCCCTGAGAAATAATCTAAGAGTTAGACAGCAGAGATAACTAG }\end{array}$ & 110 \\
\hline Af-PLP & & $\begin{array}{l}\text { P-CCTGCAACCCCCAATTTCCATTCCCAATAGGTCCAGAATGTCAGCCGTTCC } \\
\text { TCACACCAGACTGCCCTGAGAAATAATC TAAGAAGAGCAAAGCTATACAAGC }\end{array}$ & 103 \\
\hline Apa-PLP & & $\begin{array}{l}\text { P-ACTTGATGTTGCTCCTGGCATTGATTTCCCAATAGGTCCAGAATGTCAGCCGTTCCT } \\
\text { CACACCAGACTGCCCTGAGAAATAATCTAAGAGGTTAGACAGCAGAGACC }\end{array}$ & 107 \\
\hline Aph-PLP & & $\begin{array}{l}\text { P-TTATTTGAAACAGCCTGCAACCCTTCCCAATAGGTCCAGAATGTCA } \\
\text { GCCGTTCCTCACACCAGACTGCCCTGAGAAATAATCTAAGAAAGAGAGCAAGGCTATTT }\end{array}$ & 105 \\
\hline Ar-PLP & & $\begin{array}{l}\text { P-TTGTTCATTGCTCCTGGCATTGATATTCCCAATAGGTCCAGAATGTCAGCCGTTCCT } \\
\text { CACACCAGACTGCCCTGAGAAATAATCTAAGAACAGTTAGCTGAGAGAACTG }\end{array}$ & 109 \\
\hline Asubf-PLP & & $\begin{array}{l}\text { P-TCAAGAGACCAGTCAAAAGTCTCTCATTTCCCAATAGGTCCAGA } \\
\text { ATGTCAGCCGTTCCTCACACCAGACTGCCCTGAGAAATA } \\
\text { ATCTAAGAGATTCCAATTCAAATCAAT }\end{array}$ & 110 \\
\hline Asubj-PLP & & $\begin{array}{l}\text { P-AGTGATATTGCTCCTGGCATTGATATTCCCAATAGGTCCAGAATGTCAGCCGTTCCTCACA } \\
\text { CCAGACTGCCCTGAGAAATAATCTAAGAGTTAGACAGCAGAGAGAAGT }\end{array}$ & 109 \\
\hline Asubp-PLP & & $\begin{array}{l}\text { P-GTTAGACAGCAGAGAGAACTAACATGGCTTCCCAATAGGTCCAG } \\
\text { AATGTCAGCCGTTCCTCACACCAGACTGCCCTGAGAAATA } \\
\text { ATCTAAGATTTTACAGACAACTGTGAGA }\end{array}$ & 112 \\
\hline Av-PLP & & $\begin{array}{l}\text { P-GTTAGACAGCAGAGAGAACTAACATGGCTTCCCAATAGGTCCAGAATGTCAGCCGTT } \\
\text { CCTCACACCAG ACTGCCCTGAGAAATAATCTAAGATTACAGACAACTGTTAGCG }\end{array}$ & 111 \\
\hline HRCA-primer 1 & & GTGAGGAACGGCTGACATTCTG & 22 \\
\hline HRCA-primer 2 & & ACCAGACTGCCCTGAGAAATAAT & 23 \\
\hline
\end{tabular}

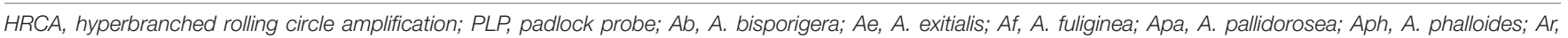

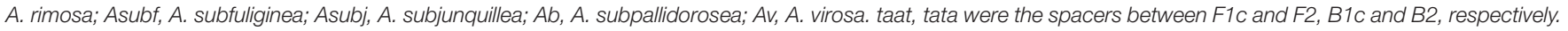
$P$ represents phosphorylation and bold represents the $5^{\prime}$ and $3^{\prime}$ arms of the PLP matched to the target region.

extracted from A. bisporigera, A. exitialis, A. fuliginea, A. pallidorosea, A. phalloides, A. rimosa, A. subfuliginea, A. subjunquillea, A. subpallidorosea, and A. virosa was used for cross reaction testing.

For the specificity of the universal primers, genomic DNA from twenty-six Amanita species listed in Table $\mathbf{1}$ was tested by the LAMP method.

\section{Sensitivity of LAMP and HRCA}

To determine the detection limit, the LAMP and HRCA assays were performed using a 10 -fold dilution series of genomic DNA from $A$. fuliginea ranging from $10 \mathrm{ng}$ to $10 \mathrm{fg}$.

\section{RESULTS}

\section{Specificity of LAMP and HRCA}

Genomic DNA from ten lethal Amanita mushrooms was used to test the specificity of the corresponding sets of specific LAMP primers and specific HRCA PLPs.

As shown in Figure 3A, the LAMP reactions were analyzed by HNB dye staining and agarose gel electrophoresis. The results of the two detection methods were consistent. Positive reactions were observed with a sky-blue mixture and typical ladder-like banding, whereas for the negative reactions, the color of the tubes remained violet, and no bands were detected after electrophoresis. The six primer sets, Ae-primers, Af-primers Ar-primers, Asubf-primers, Asubp-primers and
Av-primers, could clearly recognize and distinguish the expected Amanita species. However, cross reaction occurred between the Ab-primers and Apa-primers and the Aph-primers and Asubj-primers.

For the HRCA, amplification products were detected by SYBR Green I dye staining and agarose gel electrophoresis. Positive HRCA results generated a typical ladder-like pattern of fragments increasing in size, comprising the monomer and multimer repeats of the amplified product formed by single and multiple copies of the circularized padlock probe, while negative reactions had a clean background. The HRCA signal was also determined by adding SYBR Green I dye after the reactions; positive reactions turned green while negative reactions remained orange. From Figure 3B, it could be seen that the probes could specifically detect their corresponding targets, and no false-positive reaction was observed. The results from analysis with SYBR Green I dye were compatible with those obtained with electrophoresis.

In addition, the phylogenetic relationship of the lethal Amanita species based on ITS sequences was analyzed, and the resulting tree (Figure 4) strongly resolved the examined taxa into seven clades comprising ten phylogenetic species. These results are consistent with the previous results of Cai et al. (2014). A. exitialis, A. fuliginea, A. rimosa, and A. subfuliginea formed a clade alone with 99 or $100 \%$ bootstrap percentages, while $A$. bisporigera and A. pallidorosea, A. phalloides and A. subjunquillea, and A. subpallidorosea and A. virosa formed a clade but were classified into two branches with 99, 98, and $96 \%$ bootstrap, respectively. By combining the tree and the 

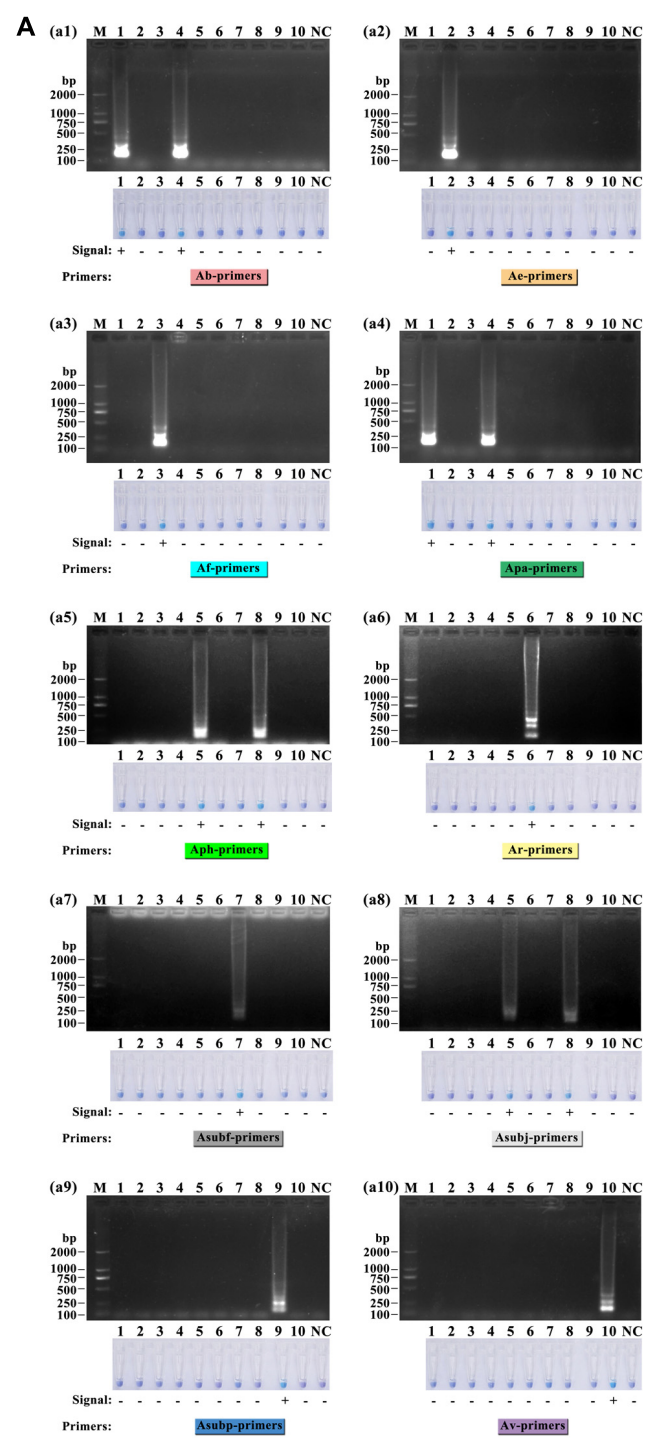
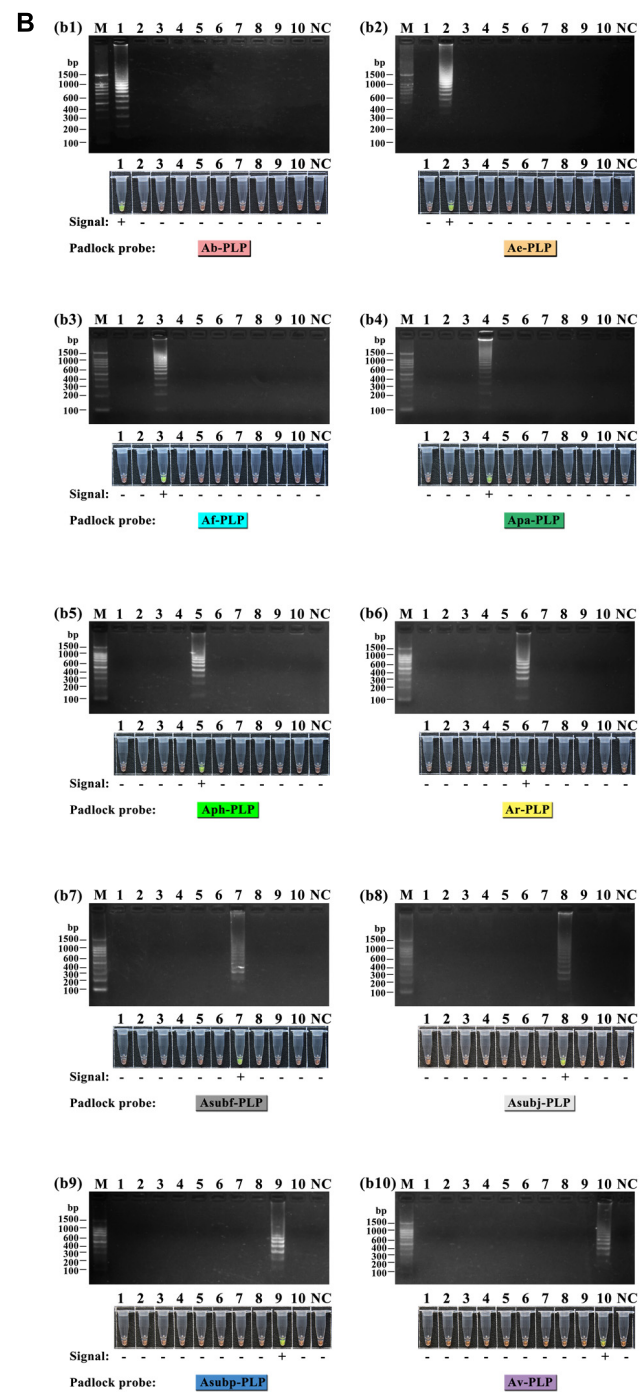

FIGURE 3 | Specificity test of the ten sets of LAMP primers (A) and ten HRCA padlock probes (B) for lethal amanitas. M: DL2000 or 100 bp ladder, 1: A. bisporigera, 2: A. exitialis, 3: A. fuliginea, 4: A. pallidorosea, 5: A. phalloides, 6: A. rimosa, 7: A. subfuliginea, 8: A. subjunquillea, 9: $A$. subpallidorosea, 10: $A$. virosa, NC: negative control.

amplification signals above, it could be intuitively found that LAMP was capable of discriminating interclade lethal Amanita species but could not perfectly discriminate the intraclade species (Clade 1 and 5 failed, Clade 3 succeeded); however, HRCA could discriminate intraclade species well. Hence, it could be concluded that the specificity of HRCA was clearly higher than LAMP.

\section{Evaluation of Universal LAMP Primers}

To verify the specificity and universality of the universal primers for lethal amanitas, the LAMP reactions were carried out with genomic DNA extracted from 10 lethal species from Amanita section Phalloideae and 16 species of Amanita outside section Phalloideae. As shown in Figure 5, the result showed that positive LAMP reaction occurred only in lethal Amanita species, while the other species were negative.

\section{Sensitivity of LAMP and HRCA}

To determine the detection limit, the LAMP reactions were performed using a serial 10-fold dilution ranging from $10 \mathrm{ng}$ to $10 \mathrm{fg}$ of DNA template of A. fuliginea. The detection limit of LAMP and HRCA were $10 \mathrm{pg}$ and $1 \mathrm{pg}$ per reaction, respectively (Figure 6). These results suggested that the detection sensitivity of HRCA was ten times higher than that of LAMP.

\section{DISCUSSION}

In the last 10 years, molecular detection based on ITS sequence has provided a promising alternative strategy for the identification of poisonous Amanita species; the ITS sequences could be used as a DNA barcode marker for lethal amanitas 


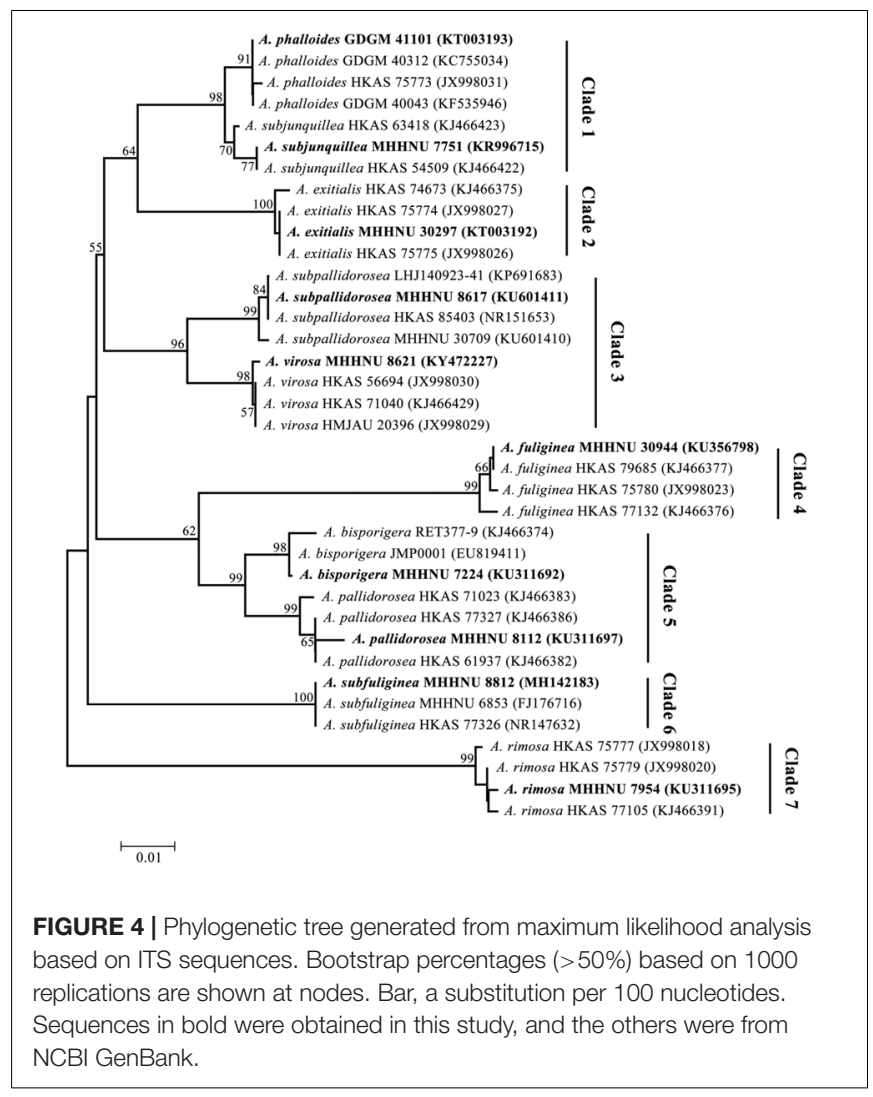

(Zhang et al., 2010; Cai et al., 2014). The phylogenetic analysis of the ITS data showed that lethal amanitas (Amanita section Phalloideae) were robustly supported as a monophyletic group, in which twenty-eight phylogenetic species were divided into nine major clades (Cai et al., 2014). Furthermore, these important molecular characteristics provide a great opportunity for us to design specific or universal primers for the rapid identification of lethal amanitas based on isothermal amplification methods. Vaagt et al. (2013) designed a set of LAMP primers based on the ITS sequence for the specific detection of death cap A. phalloides. The limited number of species of Amanita in the institute collection did not represent all species of Amanita; the related Amanita species tested in our study, such as A. muscaria, A. citrina, A. pantherina, and A. rubescens, are species outside section Phalloideae. In our present study, we endeavored to develop a series of species-specific LAMP primers capable of distinguishing each lethal amanitas within section Phalloideae. The results showed that the LAMP-based method could distinguish available interclade Amanita species but mostly failed to distinguish intraclade Amanita species. Some lethal Amanita species are very closely evolutionarily related based on small variations in ITS sequences, which are highly similar and identical (Zhang et al., 2010; Cai et al., 2014, 2016). For example, for A. bisporigera and A. pallidorosea, their ITS are almost the same, with a $98 \%$ identity, and they are in the same clade but classified into two branches in the phylogenetic tree (Figure 4), which indicated that LAMP has a certain limitation, and the specificity of the method is not applicable for highly identical templates. Indeed, it was reported that SNP-LAMP was developed to detect allele specific detection or single nucleotide polymorphisms (Fukuta et al., 2006; Ayukawa et al., 2017; Yongkiettrakul et al., 2017). However, it should be noted that some objective factors, such as the base composition of the target template, SNP distribution and amount, melting temperature and GC content of the primer, could affect the final result of SNPLAMP detection. As reported, Yongkiettrakul et al. (2017) failed to distinguished between the wild-type and quadruple mutant dhfr gene of Plasmodium falciparum by SNP-LAMP. In our study, many attempts were made to design SNP-LAMP primers for intraclade Amanita species; however, only A. subpallidorosea and A. virosa (Clade3) were distinguished successfully, and the other two intraclade Amanita species, A. bisporigera and A. pallidorosea (Clade5) and $A$. phalloides and $A$. subjunquillea (Clade1) were not distinguished.

It has been reported hyperbranched rolling cycle amplification coupled with PLP was a particularly useful tool to discriminate closely related species and even subtypes of species with minimal nucleotide polymorphisms (Tong et al., 2007; Najafzadeh et al., 2013; Lin et al., 2018). Therefore, 10 specific PLPs were subsequently designed for each of the 10 lethal amanitas in our present experiment. The results suggested that the HRCA-based assay was able to determine whether each species of 10 lethal amanitas was interclade or intraclade. Notably, A. bisporigera was clearly distinguished from A. pallidorosea, and A. phalloides was also clearly distinguished from $A$. subpallidorosea by HRCA. Even though these two pairs failed to be distinguished by LAMP, these results indicated that HRCA had a higher specificity than LAMP. The high specificity of HRCA resulted from the single base recognition capability of the PLP, which is sensitive to mismatches between the probe and the target (Pickering et al., 2002; Szemes et al., 2005). It was confirmed that mismatches positioned at the $3^{\prime}$ end of PLP were strongly discriminating (Pickering et al., 2002; Szemes et al., 2005), which confers definite and informative target sites for detection. Next, increasing the hybridization temperature and shortening the $3^{\prime}$ arm of the PLP with melting temperature below the ligation temperature are considered to further improve specificity (Faruqi et al., 2001; van Doorn et al., 2007). According these rules, the PLPs designed in our study were preferred with more discriminating bases in the $3^{\prime}$ terminal and short $3^{\prime}$ arms, which induce extremely high specificity.

Furthermore, to distinguish the lethal Amanita species in section Phalloideae from the other Amanita species outside of section Phalloideae, a set of universal primers was designed based on the multiple alignment of thirty-six published ITS sequences of fifteen lethal Amanita species. The results showed that a positive LAMP reaction occurred only in lethal Amanita species, while the rest were negative, which indicated this LAMP method could distinguish the lethal Amanita species from the other Amanita species outside of section Phalloideae. Because these lethal Amanita species account for over 90\% of all fatal mushroom poisonings worldwide, amatoxins are the common chemical property of these Amanita species, which induce acute liver failure (Walton, 2018). In the treatment of clinical poisoning, it is sometimes more important to determine the 

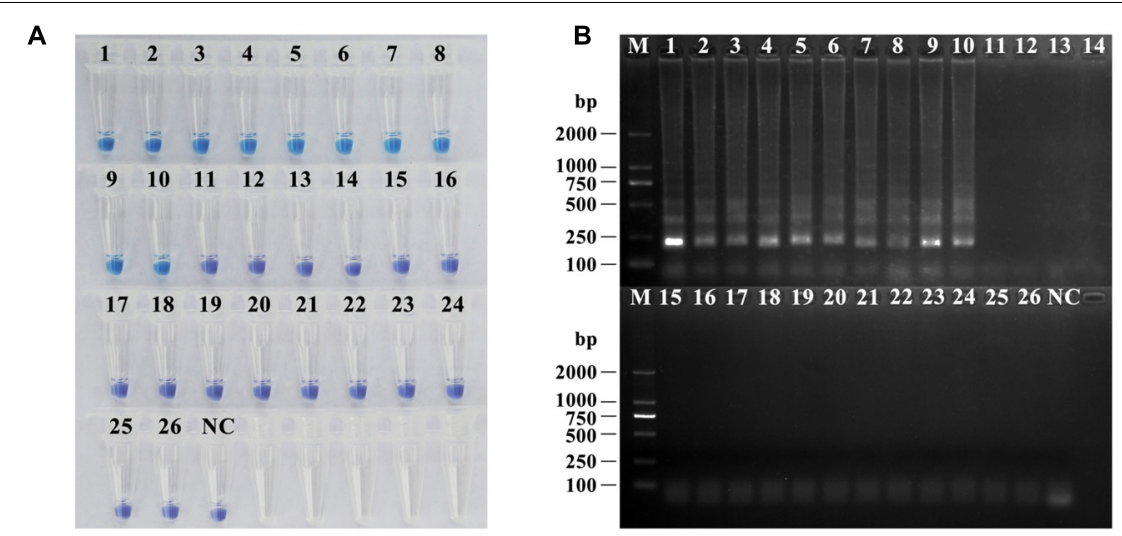

FIGURE 5 | Specificity and universality tests of the universal primers for lethal amanitas. (A) Coloration of LAMP by adding HNB dye; (B) Electrophoresis analysis of LAMP-amplified products. M: DL2000, 1: A. bisporigera, 2: A. exitialis, 3: A. fuliginea, 4: A. pallidorosea, 5: A. phalloides, 6: A. rimosa, 7: A. subfuliginea, 8: A. subjunquillea, 9: A. subpallidorosea, 10: A. virosa, 11: Amanita rubrovolvata, 12: A. rufoferruginea, 13: $A$. sinensis, 14: $A$. sychnopyramis, 15: $A$. javanica, 16: A. fulva, 17: A. orientifulva, 18: A. vaginata, 19: A. neoovoidea, 20: A. kotohiraensis, 21: A. oberwinklerana, 22: A. pseudoporphyria, 23: A. citrina 24: A. orsonii, 25: A. sepiacea, 26: A. spissacea, NC: negative control.
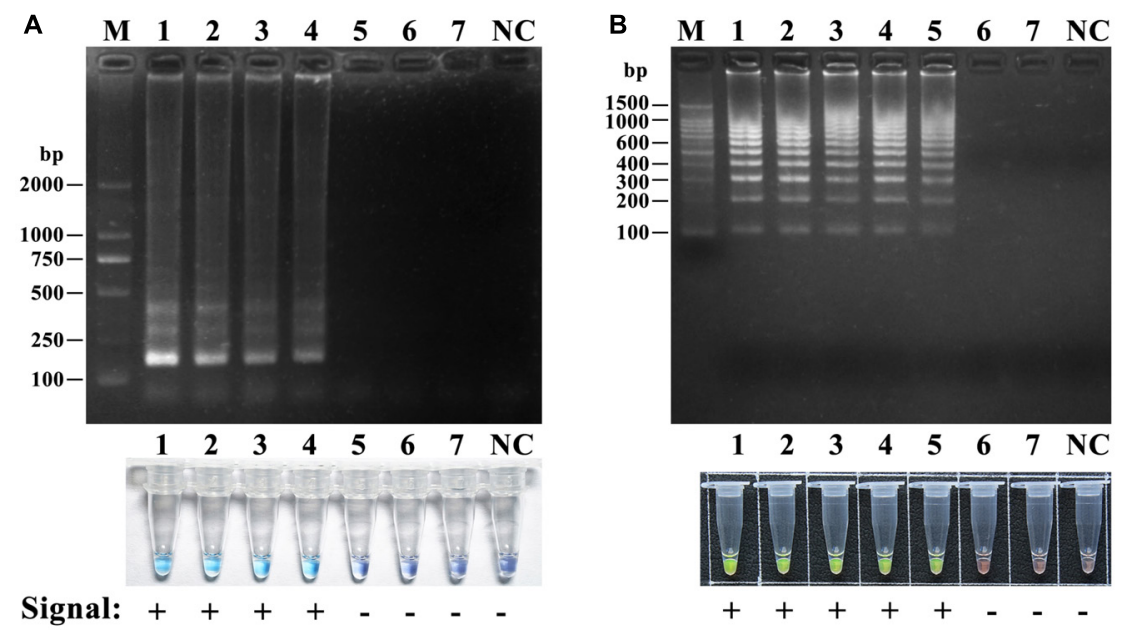

FIGURE 6 | Sensitivity of the LAMP assay (A) and the HRCA (B) assay for A. fuliginea. M: DL 2000; NC, negative control. A dilution series of $A$. fuliginea DNA was as follows: 1, $10 \mathrm{ng} ; 2,1 \mathrm{ng} ; 3,100 \mathrm{pg}, 4,10 \mathrm{pg} ; 5,1 \mathrm{pg} ; 6,100 \mathrm{fg} ; 7,10 \mathrm{fg}$.

nature of the species than to determine the accurate species; in this case, this universal LAMP method could be used to rapidly determine whether the species is lethal.

Loop-mediated isothermal amplification and HRCA possess a sensitivity advantage that is $10-100$ times higher than conventional PCR (Wang et al., 2012, 2015). Our results showed that the detection limits of the two methods could be at the pg level for the mushroom genomic DNA, and the sensitivity of HRCA was 10 times higher than that of LAMP, which was consistent with Wang et al. (2012). Compared to HRCA, the LAMP detection test was more rapid and simple, where white precipitate was generated by the naked eye within approximately $1 \mathrm{~h}$. However, this technique had a very high risk for contamination, and the precipitate was inconveniently observational. Therefore, hydroxyl naphthol blue (HNB) was used as an indicative dye for the LAMP reaction in this study.
When HNB was added before the reaction, a positive reaction will produce large amounts of magnesium pyrophosphate precipitate, thus producing $\mathrm{Mg}^{2+}$ and a $\mathrm{pH}$ change, and the color of the reaction solution change from violet into blue, so the result is easy to observe, and it does not cause aerosol pollution without opening the tube (Goto et al., 2009). In contrast, HRCA was relatively complicated and needed hours for completion for the extra PLP ligation and exonucleolysis steps. Nevertheless, we proved that exonucleolysis could be omitted because background signals caused by linear probes were almost invisible and insusceptible (data not shown), as was also found by Lackner et al. (2012) and Lin et al. (2018). Thus, the exclusion of an exonuclease reaction shortened the procedure by at least $2 \mathrm{~h}$, and HRCA detection could be completed within $2 \mathrm{~h}$ (an hour for PLP cyclization and another hour for amplification) in our study. Despite more reagents and procedures, HRCA is more 
specific and sensitive than LAMP as described earlier. PCR amplification and sequencing of the ITS is the gold standard for mushroom species identification. However, compared with the two isothermal amplification methods above PCR-based method requires the expensive instrument for thermal cycling and extra time and cost for gel electrophoresis and sequencing and the species identification period using sequencing of ITS usually takes 1-2 working day. But for LAMP and HRCA, the identification only requires a water bath for the reaction and the detection can be completed and judged by dye staining within several hours. Therefore, LAMP and HRCA detection are rapider and require lower cost than PCR.

In conclusion, the LAMP and HRCA-based assays established in this study provided rapid, specific, sensitive and cost-effective tools for the detection and identification of lethal amanitas.

\section{DATA AVAILABILITY}

All datasets generated for this study are included in the manuscript and/or the Supplementary Files.

\section{AUTHOR CONTRIBUTIONS}

ZC conceived and designed the experiments. ZH, YS, and SL carried out the LAMP and HRCA assay. PL carried

\section{REFERENCES}

Ayukawa, Y., Hanyuda, S., Fujita, N., Komatsu, K., and Arie, T. (2017). Novel loop-mediated isothermal amplification (LAMP) assay with a universal QProbe can detect SNPs determining races in plant pathogenic fungi. Sci. Rep. 7:4253. doi: 10.1038/s41598-017-04084-y

Cai, Q., Cui, Y. Y., and Yang, Z. L. (2016). Lethal Amanita species in China. Mycologia 108, 993-1009. doi: 10.3852/16-008

Cai, Q., Tulloss, R. E., Tang, L. P., Tolgor, B., Zhang, P., Chen, Z. H., et al. (2014). Multi-locus phylogeny of lethal amanitas: implications for species diversity and historical biogeography. BMC Evol. Biol. 14:143. doi: 10.1186/1471-214814-143

Chen, Z. H., Zhang, P., and Zhang, Z. G. (2014). Investigation and analysis of 102 mushroom poisoning cases in Southern China from 1994 to 2012. Fungal Divers. 64, 123-131. doi: 10.1007/s13225-013-0260-7

Dai, T. T., Lu, C. C., Lu, J., Dong, S., Ye, W., Wang, Y., et al. (2012). Development of a loop-mediated isothermal amplification assay for detection of Phytophthora sojae. FEMS Microbiol. Lett. 334, 27-34. doi: 10.1111/j.1574-6968.2012. 02619.x

Davari, M., Diepeningen, A. D. V., Babai-Ahari, A., Arzanlou, M., Najafzadeh, M. J., Lee, T. A., et al. (2012). Rapid identification of Fusarium graminearum species complex using rolling circle amplification (RCA). J. Microbiol. Meth. 89, 63-70. doi: 10.1016/j.mimet.2012.01.017

Duan, Y. B., Ge, C. Y., Zhang, X. K., Wang, J. X., and Zhou, M. G. (2014). Development and evaluation of a novel and rapid detection assay for Botrytis cinerea based on loop-mediated isothermal amplification. PLoS One 9:e111094. doi: 10.1371/journal.pone.0111094

Enjalbert, F., Gallion, C., Jehl, F., Monteil, H., and Faulstich, H. (1992). Simultaneous assay for amatoxins and phallotoxins in Amanita phalloides Fr. by high-performance liquid chromatography. J. Chromatogr. 598, 227-236. doi: 10.1016/0021-9673(92)85052-U

Enjalbert, F., Rapior, S., Nouguier-Soulé, J., Guillon, S., Amouroux, N., and Cabot, C. (2002). Treatment of amatoxin poisoning: 20-year retrospective analysis. J. Toxicol. clin. Toxic. 40, 715-757. doi: 10.1081/CLT-120014646 out the analysis of ITS DNA sequences of all species and phylogenetic tree building. $\mathrm{PZ}$ provided some Amanita materials and identified the species. $\mathrm{ZH}$ and $\mathrm{ZC}$ wrote the manuscript.

\section{FUNDING}

This study was supported by the National Natural Science Foundation of China (Grant No. 31872616), Hunan Provincial Construct Program of the Key Discipline in Ecology, China (Grant No. 0713), and Hunan Provincial Cooperative Innovation Center of Engineering and New Products for Developmental Biology, China (Grant No. 20134486).

\section{SUPPLEMENTARY MATERIAL}

The Supplementary Material for this article can be found online at: https://www.frontiersin.org/articles/10.3389/fmicb. 2019.01523/full\#supplementary-material

FIGURE S1 | Basidiomata of nine lethal Amanita species. (A) A. bisporigera (MHHNU 7224); (B) A. exitialis (MHHNU 30297); (C) A. fuliginea (MHHNU 30944); (D) A. pallidorosea (MHHNU 8112); (E) A. rimosa (MHHNU 7954); (F) A. subfuliginea (MHHNU 8812); (G) A. subjunquillea (MHHNU 7751); (H) A. subpallidorosea (MHHNU 8617); (I) A. virosa (MHHNU 8621; photos A, D, E, F, $\mathrm{G}$, and $\mathrm{H}$ by $\mathrm{PZ}$; photos $\mathrm{B}, \mathrm{C}$, and I by ZC).

Epis, S., Matinato, C., Gentili, G., Varotto, F., Bandi, C., and Sassera, D. (2010). Molecular detection of poisonous mushrooms in different matrices. Mycologia 102, 747-754. doi: 10.3852/09-124

Faruqi, A. F., Hosono, S., Driscoll, M. D., Dean, F. B., Alsmadi, O., Bandaru, R., et al. (2001). High-throughput genotyping of single nucleotide polymorphisms with rolling circle amplification. BMC Genomics 2:4. doi: 10.1186/14712164-2-4

Fukuta, S., Mizukami, Y., Ishida, A., and Kanbe, M. (2006). Development of loopmediated isothermal amplification (LAMP)-based SNP markers for shelf-life in melon (Cucumis melo L.). J. Appl. Genet. 47, 303-308. doi: 10.1007/BF03194639

Goto, M., Honda, E., Oqura, A., Nomoto, A., and Hanaki, K. (2009). Clorimetric detection of loop-mediated isothermal amplification reaction by using hydroxy naphthol blue. Biotechniques 46, 167-172. doi: 10.2144/000113072

Harper, K. A., Smart, C. D., and Davis, R. M. (2011). Development of a DNA-based macroarray for the detection and identification of Amanita species. J. Forensic Sci. 56, 1003-1009. doi: 10.1111/j.1556-4029.2011.01739.x

Kaocharoen, S., Wang, B., Tsui, K. M., Trilles, L., Kong, F., and Meyer, W. (2008). Hyperbranched rolling circle amplification as a rapid and sensitive method for species identification within the Cryptococcus species complex. Electrophoresis 29, 3183-3191. doi: 10.1002/elps.200700903

Lackner, M., Najafzadeh, M. J., Sun, J., Lu, Q., and de Hoog, G. S. (2012). Rapid identification of Pseudallescheria and Scedosporium strains by using rolling circle amplification. Appl. Environ. Microb. 78, 126-133. doi: 10.1128/AEM. 05280-11

Larkin, M. A., Blackshields, G., Brown, N. P., Chenna, R., Mcgettigan, P. A., Mcwilliam, H., et al. (2007). Clustal W and Clustal X version 2.0. Bioinformatics 23, 2947-2948. doi: 10.1093/bioinformatics/btm404

Lin, H., Jiang, X., Yi, J., Wang, X., Zuo, R., Jiang, Z., et al. (2018). Molecular identification of Neofabraea species associated with bull's eye rot on apple using

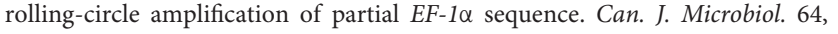
57-68. doi: 10.1139/cjm-2017-0448

Lizardi, P. M., Huang, X., Zhu, Z., Bray-Ward, P., Thomas, D. C., and Ward, D. C. (1998). Mutation detection and single-molecule counting using isothermal rolling-circle amplification. Nat. Genet. 19, 225-232. doi: 10.1038/898 
Nagamine, K., Hase, T., and Notomi, T. (2002). Accelerated reaction by loopmediated isothermal amplification using loop primers. Mol. Cell. Probe. 16, 223-229. doi: 10.1006/mcpr.2002.0415

Najafzadeh, M. J., Dolatabadi, S., Keisari, M. S., Naseri, A., Feng, P., and de Hoog, G. S. (2013). Detection and identification of opportunistic Exophiala species using the rolling circle amplification of ribosomal internal transcribed spacers. J. Microbiol. Meth. 94, 338-342. doi: 10.1016/j.mimet.2013.06.026

Niessen, L., and Vogel, R. F. (2010). Detection of Fusarium graminearum DNA using a loop-mediated isothermal amplification (LAMP) assay. Int. J. Food Microbiol. 140, 183-191. doi: 10.1016/j.ijfoodmicro.2010.03.036

Nilsson, M., Malmgren, H., Samiotaki, M., Kwiatkowski, M., Chowdhary, B. P., and Landegren, U. (1994). Padlock probes: circularizing oligonucleotides for localized DNA detection. Science 265, 2085-2088. doi: 10.1126/science.7522346

Notomi, T., Okayama, H., Masubuchi, H., Yonekawa, T., Watanabe, K., Amino, N., et al. (2000). Loop-mediated isothermal amplification of DNA. Nucleic Acids Res. 28:E63. doi: 10.1097/RLU.0b013e3181f49ac7

Pickering, J., Bamford, A., Godbole, V., Briggs, J., Scozzafava, G., Roe, P., et al. (2002). Integration of DNA ligation and rolling circle amplification for the homogeneous, end-point detection of single nucleotide polymorphisms. Nucleic Acids Res. 30:e60. doi: 10.1093/nar/gnf060

Szemes, M., Bonants, P., Weerdt, M., Baner, J., Landegren, U., and Schoen, C. D. (2005). Diagnostic application of padlock probes-multiplex detection of plant pathogens using universal microarray. Nucleic Acids Res. 33:e70. doi: 10.1093/ nar/gni069

Tamura, K., Stecher, G., Peterson, D., Filipski, A., and Kumar, S. (2013). MEGA6: molecular evolutionary genetics analysis Version 6.0. Mol. Biol. Evol. 30, 2725-2729. doi: 10.1093/molbev/mst197

Tang, S. S., Zhou, Q., He, Z. M., Luo, T., Zhang, P., Cai, Q., et al. (2016). Cyclopeptide toxins of lethal amanitas: compositions, distribution and phylogenetic implication. Toxicon 120, 78-88. doi: 10.1016/j.toxicon.2016. 07.018

Tong, Z., Kong, F., Wang, B., Zeng, X., and Gilbert, G. L. (2007). A practical method for subtyping of Streptococcus agalactiae serotype III, of human origin, using rolling circle amplification. J. Microbiol. Meth. 70, 39-44. doi: 10.1016/j.mimet. 2007.03.010

Trilles, L., Wang, B., Firacative, C., Lazéra, M. S., Wanke, B., and Meyer, W. (2014). Identification of the major molecular types of Cryptococcus neoformans and C. gattii by Hyperbranched rolling circle amplification. PLoS One 9:e94648. doi: 10.1371/journal.pone.0094648

Tsui, C. K., Wang, B., Khadempour, L., Alamouti, S. M., Bohlmann, J., Murray, B. W., et al. (2010). Rapid identification and detection of pine pathogenic fungi associated with mountain pine beetles by padlock probes. J. Microbiol. Meth. 83, 26-33. doi: 10.1016/j.mimet.2010.07.016
Vaagt, F., Haase, I., and Fischer, M. (2013). Loop-mediated isothermal amplification LAMP)-based method for rapid mushroom species identification. J. Agr. Food Chem. 61, 1833-1840. doi: 10.1021/jf304824b

van Doorn, R., Szemes, M., Bonants, P., Kowalchuk, G. A., Salles, J. F., Ortenberg, E., et al. (2007). Quantitative multiplex detection of plant pathogens using a novel ligation probe-based system coupled with universal, high-throughput real-time PCR on OpenArrays ${ }^{\text {TM }}$. BMC Genomics 8:276. doi: 10.1186/14712164-8-276

Walton, J. (2018). "Biological activities of the Amanita peptide toxins," in The Cyclic Peptide Toxins of Amanita and Other Poisonous Mushrooms, ed. J. Walton (Cham: Springer-Verlag Press), 131-142.

Wang, X., Teng, D., Tian, F., Guan, Q., and Wang, J. (2012). Comparison of three DNA extraction methods for feed products and four amplification methods for the 5'-junction fragment of Roundup Ready soybean. J. Agr. Food Chem. 60, 4586-4595. doi: 10.1021/jf300827q

Wang, X. R., Wu, L. F., Wang, Y., Ma, Y. Y., Chen, F. H., and Ou, H. L. (2015). Rapid detection of Staphylococcus Aureus by loop-mediated isothermal amplification. Appl. Biochem. Biotech. 175, 882-891. doi: 10.1007/s12010-0141328- $\mathrm{x}$

Wieland, T. (1986). Peptides of Poisonous Amanita Mushrooms. New York, NY: Springer-Velag Press.

Yongkiettrakul, S., Kampeera, J., Chareanchim, W., Rattanajak, R., Pornthanakasem, W., Kiatpathomchai, W., et al. (2017). Simple detection of single nucleotide polymorphism in Plasmodium falciparum by SNPLAMP assay combined with lateral flow dipstick. Parasitol. Int. 66, 964-971. doi: 10.1016/j.parint.2016.10.024

Zhang, P., Chen, Z. H., Xiao, B., Tolgor, B., Bao, H. Y., and Yang, Z. L. (2010). Lethal amanitas of East Asia characterized by morphological and molecular data. Fungal Divers. 42, 119-133. doi: 10.1007/s13225-010-0018-4

Zhou, J., Yuan, Y., Lang, N., Yin, Y., and Sun, C. Y. (2016). Analysis of hazard in mushroom poisoning incidents in China mainland. Chin. J. Emerg. Med. 25, 724-728. doi: 10.3760/cma.j.issn.1671-0282.2016.06.008

Conflict of Interest Statement: The authors declare that the research was conducted in the absence of any commercial or financial relationships that could be construed as a potential conflict of interest.

Copyright (C) $2019 \mathrm{He}$, Su, Li, Long, Zhang and Chen. This is an open-access article distributed under the terms of the Creative Commons Attribution License (CC BY). The use, distribution or reproduction in other forums is permitted, provided the original author(s) and the copyright owner(s) are credited and that the original publication in this journal is cited, in accordance with accepted academic practice. No use, distribution or reproduction is permitted which does not comply with these terms. 